\title{
Retrospective Case Series of Aripiprazole Augmentation in Pervasive Developmental Disorders
}

\author{
Yeni Kim*, Soo-Churl Cho, Min-Sup Shin, Jae-Won Kim, Seung-Hee Lee ${ }^{\dagger}$ and Boong-Nyun Kim ${ }^{凶}$ \\ Division of Child \& Adolescent Psychiatry, Department of Psychiatry and Institute of Human Behavioral Medicine, Seoul National University \\ College of Medicine, Seoul, Korea
}

Due to co-morbidities and treatment resistant nature of pervasive developmental disorder (PDD), diverse combinations of regimens have been tried. This retrospective study aimed to explore adjunctive use of aripiprazole in children with PDD. Changes in illness severity were measured by Clinical Global Impression of Severity (CGI-S) and Clinical Global Impression of Improvement (CGI-I) in 14 aripiprazoletreated patients with PDD. Improvement of illness severity was observed after aripiprazole add-on ( $5.8 \pm 0.8$ to $4.9 \pm 1.0, \mathrm{Z}=-2.75$, $\mathrm{p}=0.001$ ). Mean dosage was $7.7 \mathrm{mg}$ /day [standard deviation (SD) 3.3, range 5-15]. A higher mean dosage was observed in group with improvement in symptoms $(\mathrm{t}=-2.33, \mathrm{df}=12, \mathrm{p}=0.004)$. The target symptoms most effectively improved after using aripiprazole were positive psychotic symptoms (mean CGI-I: $2.0 \pm 1.4,3$ responders/4 patients, $75 \%$ response) followed by aggressive behavior $(2.5 \pm 1.7,3 / 4,75 \%)$, self-injurious behavior $(2.0 \pm 1.0,2 / 3,67 \%)$, stereotypic behavior $(2.7 \pm 1.2,2 / 3,67 \%)$, tic $(2.8 \pm 1.0,2 / 4,50 \%)$, irritability $(3.5 \pm 2.1,1 / 2,50 \%)$, obsessive behavior $(2.5 \pm 2.1,1 / 3,33 \%)$, hyperactivity $(3.4 \pm 1.6,3 / 7,43 \%)$ and mood fluctuation $(3,0 / 1$, no response). Five patients (35\%) discontinued aripiprazole due to treatment-emergent adverse effects (akathisia, insomnia, withdrawal). The results of this study suggest that aripiprazole augmentation may be used safely in maladaptive behaviors of some populations of PDD. However, future studies are required to confirm these preliminary findings.

Psychiatry Investig 2010;7:220-223

Key Words Aripiprazole, Augmentation, Autism, Pervasive developmental disorder.

\section{INTRODUCTION}

Pervasive developmental disorder (PDD) is a challenging psychiatric condition characterized by impairment in social interactions, deficits in communication and challenges with stereotypic behaviors. ${ }^{1}$ Children with PDD often display severe maladaptive behaviors, including aggression, hyperactivity, and difficulties with eating and sleeping. ${ }^{1}$ Although, no specific pharmacologic treatment exists for the core symptoms of PDD, medication serves as one of the mainstays of treatment in ameliorating the associated maladaptive behavioral symptoms of PDD. ${ }^{1}$

Aripiprazole is a novel antipsychotic, which shows partial agonist effects at D2 and serotonin 5-HT1A receptors. ${ }^{2}$ Aripipra-

Received: December 11, 2009 Revised: April 1, 2010

Accepted: April 23, 2010 Available online: July 9, 2010

$\triangle$ Correspondence: Boong-Nyun Kim, MD, PhD

Division of Child \& Adolescent Psychiatry, Department of Psychiatry and Institute of Human Behavioral Medicine, Seoul National University College of Medicine, 101 Daehak-ro, Jongno-gu, Seoul 110-744, Korea

Tel: +82-2-2072-3647, Fax: +82-2-747-2471, E-mail: kbn1@snu.ac.kr

*Yeni Kim, move to Department of Adolescent Psychiatry, Seoul National Hospital, Seoul, Korea

†Seung-Hee Lee, move to Junior Mind Clinic Didim Secho, Seoul, Korea

(a) This is an Open Access article distributed under the terms of the Creative Commons Attribution Non-Commercial License (http://creativecommons.org/licenses/bync/3.0) which permits unrestricted non-commercial use, distribution, and reproduction in any medium, provided the original work is properly cited. zole was associated with a better metabolic profile compared to previous antipsychotics. A randomized control trial in children and adolescents with schizophrenia, ${ }^{3}$ reported no significant increase in weight, body mass index or other metabolic parameters including cholesterol, fasting glucose, triglycerides, and one open label trial reported a significant weight loss when changing from other atypical antipsychotics to aripiprazole. ${ }^{4}$

With its unique receptor profile, aripiprazole is being tried as augmentation for variety of treatment refractory psychiatric conditions. ${ }^{5}$ In this study, we report a retrospective case series of aripiprazole-treated PDD patients receiving other psychotropic medications in a homogenous sample of Asian ethnicity.

\section{METHODS}

Patient records from the Child and Adolescent Psychiatric Unit at Seoul National University Children's Hospital from April 2004 to December 2007 were reviewed. Fourteen patients diagnosed with autism, Asperger's disorder or PDD according to DSM-IV criteria were identified as receiving aripiprazole augmentation therapy.

Baseline and endpoint measures of the total illness severity were assessed by the Clinical Global Impression of Severity 
of Illness (CGI-S) score. Improvement in target symptoms, focusing on aggression, self-injury, stereotypy, tics, obsessive behavior, and hyperactivity, were determined by the Clinical Global Impression of Improvement (CGI-I). Patients showing the endpoint CGI-I rating of 1 or 2 were considered "responders". Two independent board-certified psychiatrists (C.S.C and L.S.H) with good inter-rater reliability $(\mathrm{K}=0.84)$ conducted the CGIs on all patients and were blind to each other's ratings. A third psychiatrist (Y.N.K) compared the two ratings. Tolerability of aripiprazole augmentation was assessed by documented adverse events. Wilcoxon's signed-rank test, Fisher's exact test and Student's t-test were used to analyze the data. All tests were 2tailed and statistical significance was set at 0.05 . The study protocol was approved by the Institutional Review Board.

\section{RESULTS}

\section{Participants}

The demographic and clinical data of each patient are listed in Table 1. The mean age was 12.1 years (range 7-17 years). Mean length of aripiprazole usage was 183.4 days. The average starting dosage was $6.1 \mathrm{mg} /$ day [standard deviation (SD) 1.9, range 5-10], and the mean final dosage was $7.7 \mathrm{mg} /$ day (SD 3.3, range 5-15). The most common reason for augmentation with aripiprazole was the lack of target symptom improvement with previous medication ( 9 out of 14 children, 64\%). In two cases, aripiprazole was added due to excessive appetite and weight gain while using other atypical antipsychotics (risperidone, olanzapine).

\section{Response}

Ten of 14 patients (71\%) showed a reduction in CGI-S scores after augmentation with aripiprazole. The CGI-S scores were significantly lower at the endpoint than at baseline $(5.8 \pm 0.8$ to $4.9 \pm 1.0$, Wilcoxon's signed rank test: $\mathrm{Z}=-2.754$, $\mathrm{p}=0.01$, effect size $=-0.74)$. The target symptoms most effectively improved after using aripiprazole were positive psychotic symptoms (mean CGI-I: $2.0 \pm 1.4,3$ responders/4 patients, $75 \%$ response) followed by aggressive behavior $(2.5 \pm 1.7,3 / 4,75 \%)$, self-injurious behavior $(2.0 \pm 1.0,2 / 3,67 \%)$, stereotypic behavior $(2.7 \pm$ $1.2,2 / 3,67 \%)$, tic $(2.8 \pm 1.0,2 / 4,50 \%)$, irritability $(3.5 \pm 2.1,1 / 2$, $50 \%)$, hyperactivity $(3.4 \pm 1.6,3 / 7,43 \%)$, obsessive behavior (2.5 $\pm 2.1,1 / 3,33 \%)$, and mood fluctuation (3, $0 / 1$, no response).

A significantly higher mean dosage was found in the response group (responders $=10.6 \mathrm{mg} /$ day, non-responders $=6$ $\mathrm{mg} /$ day; $\mathrm{t}=-2.33, \mathrm{df}=12, \mathrm{p}=0.04)$. Five $(71 \%)$ of seven patients with mental retardation and four (57\%) of seven patients without mental retardation responded to aripiprazole (Fisher's exact test, $\mathrm{p}>0.99)$. Further analysis showed that neither age $(t=-0.56$, $\mathrm{df}=9, \mathrm{p}=0.59)$ nor duration of treatment $(\mathrm{t}=-1.33, \mathrm{df}=12, \mathrm{p}=$ $0.21)$ significantly affected the patients' responses to the drug.

\section{Tolerability}

At the time of data collection, eight of the 14 (57\%) patients remained on aripiprazole treatment. Aripiprazole was discontinued in five patients due to unacceptable adverse effects (akathisia, insomnia and withdrawal) and was stopped in one patient due to target symptom (hyperactivity and tic) improvement. The mean duration of aripiprazole usage until discontinuation was 75.4 days (SD 44.2). Adverse events reported by the patients included akathisia $(n=2,14.3 \%)$, withdrawal $(n=2$, $14.3 \%)$, insomnia $(n=2,14.3 \%)$, dullness $(n=1,7.1 \%)$, increased masturbation $(n=1,7.1 \%)$, skin rash $(n=1,7.1 \%)$, dizziness $(n=1$, $7.1 \%)$, and vomiting $(\mathrm{n}=1,7.1 \%)$.

\section{Aripiprazole and concomitant medication}

The response and tolerability of aripiprazole according to the concomitant medication was analyzed. Of the seven patients using risperidone concomitantly with aripiprazole, five patients (71\%) showed improvement in CGI-S and three patients (43\%) discontinued aripiprazole during the trial. For three patients using valproic acid or divalproex concomitantly with aripiprazole, three patients (100\%) showed improvement and one patient (33\%) discontinued aripiprazole. For three patients using fluoxetine concomitantly with aripiprazole, three patients (100\%) showed improvement and one patient (33\%) discontinued aripiprazole. For three patients using mirtazapine concomitantly with aripiprazole, two patients (67\%) showed improvement while two patients (67\%) discontinued aripiprazole. For two patients on methylphenidate, one patient (50\%) showed improvement while one patient (50\%) discontinued the aripiprazole.

\section{DISCUSSION}

The dosage used in this study for augmentative effects was $7.7 \mathrm{mg} /$ day (range 5-15 mg/day). This is comparable to the dosage reported by a 14-week prospective open-label study, ${ }^{6}$ which reported mean $7.8 \mathrm{mg}$ /day (range: $2.5-15 \mathrm{mg}$ ) of aripiprazole monotherapy in 25 PDD patients aged 5-17 years. Our finding is also consistent with the results from a retrospective naturalistic study examining 34 PDD patients of 4.5-15 years, which reported the mean dosage of $8.1 \mathrm{mg} /$ day. ${ }^{7}$

The only open label prospective study published to date, reported no adverse events related to discontinuation of aripiprazole. ${ }^{6}$ However, other long-term retrospective studies reported $17-59 \%$ discontinuation rates due to adverse effects. ${ }^{8,9}$ These previously observed adverse events and subsequent discontinuation of monotherapy with aripiprazole are similar to the adverse events observed in the present study on augmentation with aripiprazole. In the present study, five of fourteen (35.7\%) children discontinued aripiprazole due to adverse events. Of the five patients who discontinued aripiprazole due to adverse 


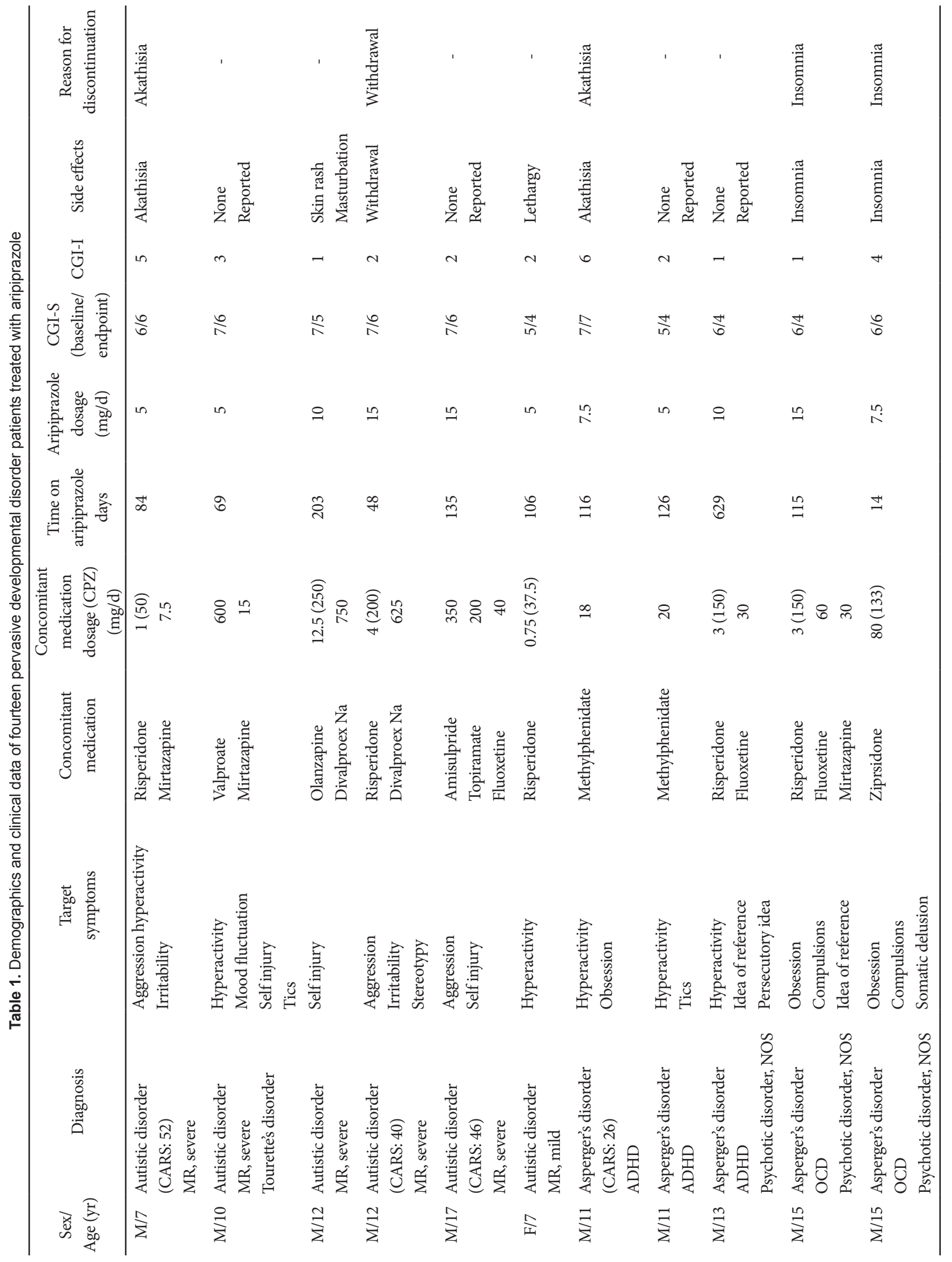




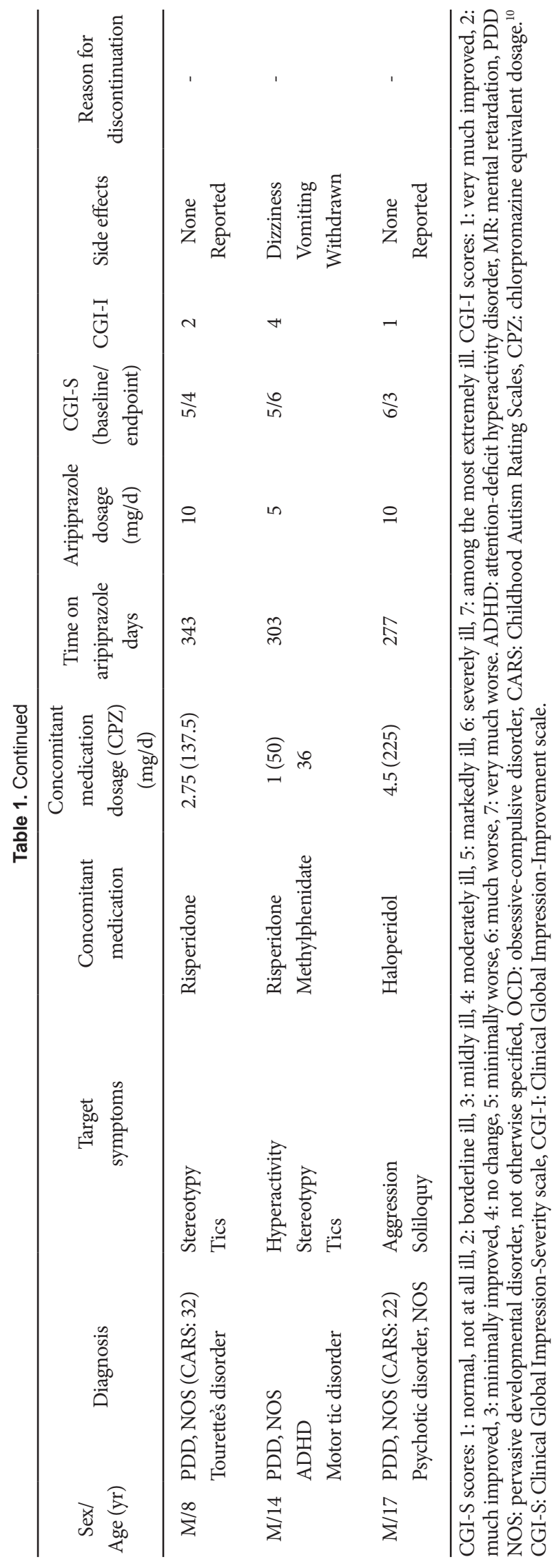

effects (akathisia and insomnia), three were concomitantly using psychotropics strongly associated with the release of dopamine (risperidone, ziprasidone, methylphenidate) or with the tendency to inhibit cytochrome P450 2D6 and 3A4 enzyme activities (fluoxetine), possibly increasing the susceptibility to adverse events. A long-term naturalistic evaluations of aripiprazole monotherapy in children and adolescents reported that 26-50\% of the patients experience adverse events. . $^{7-9}$ Adverse events occurred in $57 \%(\mathrm{n}=8)$ patients in this study suggesting that augmentation use may have similar rate of adverse event.

The CGI evaluations, although very carefully performed by two board-qualified psychiatrists, were conducted retrospectively, allowing a potential source of bias. The weight change could not be evaluated due to the concomitant usage of other psychotropics. The data, though limited by small sample size and naturalistic design, suggest that aripiprazole augmentation may be done safely for the management of maladaptive behaviors in some populations of Asian children with PDD. However, future trials are required to confirm these preliminary findings.

\section{REFERENCES}

1. National Institutes of Health. 'Autism fact sheet' NINDS. Bethesda, MD: National Institutes of Health; 2006.

2. Kikuchi T, Tottori K, Uwahodo Y, Hirose T, Miwa T, Oshiro Y, et al. 7-(4-[4-(2,3-Dichlorophenyl)-1-piperazinyl]butyloxy)-3,4-dihydro2(1H)-quinolinone (OPC-14597), a new putative antipsychotic drug with both presynaptic dopamine autoreceptor agonistic activity and postsynaptic D2 receptor antagonistic activity. J Pharmacol Exp Ther 1995;274:329-336.

3. Findling RL, Robb A, Nyilas M, Forbes RA, Jin N, Ivanova S, et al. A multi-center, randomized, double-blind, placebo-controlled study of oral aripiprazole for treatment of adolescents with schizophrenia. Am J Psychiatry 2008;165:1432-1441.

4. Stigler KA, Posy DJ, McDougle CJ. Aripiprazole for maladaptive behavior in pervasive developmental disorders. J Child Adolesc Psychopharmacol 2004;14:455-463.

5. Andersen SE, Johansson M, Manniche C. The prescribing pattern of a new antipsychotic: a descriptive study of aripiprazole for psychiatric inpatients. Basic Clin Pharmacol Toxicol 2008;103:75-81.

6. Stigler KA, Diener JT, Kohn AE, Li L, Erickson CA, Posey DJ, et al. Aripiprazole in pervasive developmental disorder not otherwise specified and Asperger's disorder: a 14-week, prospective, open-label study. J Child Adolesc Psychopharmacol 2009;19:265-274.

7. Masi G, Cosenza A, Millepiedi S, Muratori F, Pari C, Salvadori F. Aripiprazole monotherapy in children and young adolescents with pervasive developmental disorders: a retrospective study. CNS Drugs 2009;23: 511-521.

8. Rugino TA, Janvier YM. Aripiprazole in children and adolescents: clinical experience. J Child Neurol 2005;20:603-610.

9. Valicenti-McDermott MR, Demb H. Clinical effects and adverse reactions of off-label use of aripiprazole in children and adolescents with developmental disabilities. J Child Adolesc Psychopharmacol 2006;16: 549-560.

10. Woods SW. Chlorpromazine equvalent doses for the newer atypical antipsychotics. J Clin Psychiatry 2003;64:663-667. 